\title{
ON THE PSEUDO-GROUP STRUCTURE OF ANALYTIC FUNCTIONS ON AN ALGEBRA
}

\author{
H. H. JOHNSON ${ }^{1}$
}

Recently C. F. Moppert [4] introduced a new definition of algebraic analyticity for functions on an algebra to itself. The purpose of this paper is to study the pseudo-group structure of these functions, i.e., their closure under inverses, composition and restrictions of domain. For a certain class of algebras necessary and sufficient conditions are determined that the algebraic analytic functions which are local bianalytic maps form a pseudo-group. In doing this the underlying differential equations are examined and certain properties of their solutions are brought to light. In case the analytic functions form a pseudo-group, it will be a continuous pseudo-group in the sense of S. Lie and E. Cartan [1].

Let $A$ be an $n$-dimensional (nonassociative) algebra over a field $F$ which we take to be the real or complex numbers. Denoting elements of $A$ by $n$-tuples $\left(x^{1}, \cdots, x^{n}\right)=\left(x^{i}\right)$ and $\left(y^{1}, \cdots, y^{n}\right)=\left(y^{i}\right)$, let the product in $A$ be defined by

$$
\left(x^{i}\right) \otimes\left(y^{i}\right)=\left(\sum_{j, k=1}^{n} \pi_{j k}^{i} x^{j} y^{k}\right) .
$$

A function $f$ on an open set $D$ of $A$ into $A$, defined by its $F$-valued component functions $f^{k}\left(x^{1}, \cdots, x^{n}\right), k=1, \cdots, n$, is called $A$ analytic if each $f^{k}$ has continuous first partial derivatives and if there exist $F$-valued functions $\lambda^{i}\left(x^{1}, \cdots, x^{n}\right), i=1, \cdots, n$, on $D$ such that

$$
\frac{\partial f^{k}}{\partial x^{i}}=\sum_{i=1}^{n} \pi_{i j}^{k} \lambda^{i}
$$

In this paper we shall study only those algebras satisfying the following condition: for at least one $k=1, \cdots, n$ the matrix

$$
\Pi^{k}=\left(\begin{array}{c}
k \\
\pi_{i j}
\end{array}\right), \quad \quad i, j=1, \cdots, n,
$$

is nonsingular. Assume $\Pi^{1}$ is nonsingular with inverse matrix $\left(\rho^{i j}\right)$. Then

$$
\lambda^{i}=\sum_{j=1}^{n} \frac{\partial f^{1}}{\partial x^{j}} \rho^{j i},
$$

Received by the editors May 11, 1960 .

${ }^{1}$ Supported by OOR Contract DA-36-ORD-2164. 
and we are led to the system of linear homogeneous first order differential equations

$$
\frac{\partial f^{k}}{\partial x^{j}}=\sum_{i=1}^{n} \sum_{h=1}^{n} \frac{\partial f^{1}}{\partial x^{h}} \rho^{h i k} \pi_{i j}^{k}
$$

$k=2, \cdots, n ; j=1, \cdots, n$, which are necessary and sufficient that $f^{1}, \cdots, f^{n}$ define an $A$-analytic function.

Let $G$ denote the set of all $A$-analytic functions $f$ which are local bianalytic transformations between their ranges $R(f)$ and domains $D(f)$, both of which are open sets. By a local bianalytic transformation I mean a homeomorphism $f$ between the open sets $D(f)$ and $R(f)$ in which each $f^{k}\left(x^{1}, \cdots, x^{n}\right)$ has a convergent power series expansion about each point of $D(f)$, and the same for its inverse.

A collection $\mathcal{G}$ of local bianalytic transformations on Euclidean $n$-space over $F$ is a pseudo-group if it satisfies the following axioms:

(i) if $f \in \mathcal{G}$ and $U$ is an open set such that $U \cap D(f)$ is not empty, then $f \mid U \cap D(f)$, the restriction of $f$ to $U \cap D(f)$, is in $\mathcal{G}$,

(ii) the identity transformation is in $\mathrm{g}$,

(iii) if $f$ and $g$ are in $g$ and $R(f) \cap D(g)$ is not empty, then $g \circ f \mid f^{-1}(R(f) \cap D(g))$ is in $\mathrm{G}$, where o denotes functional composition,

(iv) if $f$ is in $G$, its inverse, $f^{-1}$, is in $g$.

We shall determine conditions under which $G$ is a pseudo-group.

Axion (i) is immediately satisfied, since if $f$ satisfies (1) on $D(f)$ its restriction to $D(f) \cap U$ will satisfy (1). Let

$$
\begin{array}{rlrl}
a_{j}^{k h} & =\sum_{i=1}^{n} \rho^{h i k} \pi_{i j}, & k & =2, \cdots, n, \\
h, j & =1, \cdots, n .
\end{array}
$$

Then (1) can be written

$$
\begin{aligned}
& \frac{\partial f^{k}}{\partial x^{j}}=\sum_{i=1}^{n} a_{j}^{k i} \frac{\partial f^{1}}{\partial x^{i}}, \\
& \begin{array}{l}
k=2, \cdots, n, \\
j=1, \cdots, n .
\end{array}
\end{aligned}
$$

Condition (ii) is satisfied if and only if $f^{i}=x^{i}, j=1, \cdots, n$ is in $G$, i.e., if and only if

$$
\delta_{j}^{k}=\sum_{h=1}^{n} a_{j}^{k h} \delta_{h}^{1}=a_{j}^{k 1}
$$

In order to discuss condition (iii) we shall need to use the following lemma:

Lemma. If (2) is satisfied, then for any n-tuple $\left(\phi_{1}, \cdots, \phi_{n}\right)$ of elements of $F$, except possibly those on a nowhere dense subset of Euclidean 
$n$-space over $F$, there exists an element $f$ of $G$ defined in an open neighborhood of $\left(a^{1}, \cdots, a^{n}\right)$ such that $\left(\partial f^{1} / \partial x^{j}\right)\left(a^{1}, \cdots, a^{n}\right)=\phi_{j}, j=1, \cdots, n$, where $\left(a^{1}, \cdots, a^{n}\right)$ is any point in Euclidean n-space over $F$.

Proof. The solution of the system (1') depends upon the solution of the system

$$
\sum_{h=1}^{n} a_{j}^{k h} \frac{\partial^{2} f^{1}}{\partial x^{h} \partial x^{i}}=\sum_{h=1}^{n} a_{i}^{k h} \frac{\partial^{2} f^{1}}{\partial x^{h} \partial x^{j}}, \quad \begin{aligned}
k & =2, \cdots, n, \\
i, j & =1, \cdots, n,
\end{aligned}
$$

for $f^{1}$. If $f^{1}$ satisfies this system $\left(1^{\prime}\right)$ becomes completely integrable as a system of equations in $f^{2}, \cdots, f^{n}$. (S) is equivalent to a system of exterior differential forms in $x^{i}, f^{1}$, and new variables $f_{i}^{1}, f_{i j}^{1}=f_{j i}^{1} ; i, j$ $=1, \cdots, n$, with $x^{1}, \cdots, x^{n}$ as independent variables:

$$
\begin{aligned}
& d f^{1}=\sum_{i=1}^{n} f_{i}^{1} d x^{i}, \\
& d f_{i}^{1}=\sum_{j=1}^{n} f_{i j}^{1} d x^{j},
\end{aligned}
$$

$$
\begin{array}{rlr}
\sum_{h=1}^{n} a_{j}^{k h} d f_{h i}^{1} & =\sum_{h=1}^{n} \begin{array}{c}
k h \\
a_{i}^{k h} d f_{h j}^{1},
\end{array} \\
\sum_{j=1}^{n} d f_{i j}^{1} \wedge d x^{j} & =0, & k=2, \cdots, n,
\end{array}
$$

where solutions are taken through an initial value where

$$
\sum_{h=1}^{n} a_{j}^{k h} f_{h i}^{1}=\sum_{h=1}^{n} a_{i}^{k h} f_{h j}^{1}
$$

and then because of the third system of equations in $\left(\mathbf{S}^{\prime}\right)$ this initial condition will persist in the solution.

If $\left(\mathrm{S}^{\prime}\right)$ is not involutive with respect to $x^{1}, \cdots, x^{n}$, its successive prolongations must be examined. Using the symmetry $f_{i j}^{1}=f_{\mathfrak{i n}}^{1},\left(\mathbf{S}^{\prime}\right)$ can be identified as one of the type studied in [2] where a specialization of Kuranishi's prolongation theorem [3] yields the information that $\left(\mathbf{S}^{\prime}\right)$ either has no solutions or solutions at all points except possibly on a proper subvariety which is nowhere dense. If (2) holds, $\left(\mathrm{S}^{\prime}\right)$ has a solution; consequently solutions exist at all points except possibly on a proper subvariety of the space of variables in the final prolongation. However, because of the nature of $\left(\mathbf{S}^{\prime}\right)$ it can be seen that there will be introduced in the successive prolongations no 0 forms which contain the variables $x^{i}, f_{j}^{1}$. In particular, solutions can 
be found where the $f_{j}^{1}$ have arbitrary initial values at $\left(a^{1}, \cdots, a^{n}\right)$. Thus, a point in some prolongation which is involutive can be found where $x^{1}=a^{1}, \cdots, x^{n}=a^{n}$.

We now raise the question whether solutions to $\left(1^{\prime}\right)$ will possess inverses. That is, for a given $n$-tuple $\partial f^{1} / \partial x^{1}, \cdots, \partial f^{1} / \partial x^{n}$, will $\left(1^{\prime}\right)$ yield a matrix $\left(\partial f^{i} / \partial x^{i}\right)$ which is singular? Since the identity belongs in $G$, the coefficients $a_{j}^{k h}$ do not impose this condition on the Jacobians, and we may deduce that except possibly for a nowhere dense set of points $\left(\phi_{1}^{1}, \cdots, \phi_{n}^{1}\right)$ the matrix $\left(\phi_{j}^{i}\right)$, where

$$
\begin{aligned}
& \phi_{j}^{k}=\sum_{h=1}^{n} a_{j}^{k h} \phi_{h}^{1}, \\
& k=2, \cdots, n \text {, } \\
& j=1, \cdots, n \text {, }
\end{aligned}
$$

is nonsingular. Thus it is seen that except possibly for $\left(\phi_{1}, \cdots, \phi_{n}\right)$ on a nowhere dense subset, the $A$-analytic function obtained will be in $G$ if restricted to a sufficiently small neighborhood of $\left(a^{1}, \cdots, a^{n}\right)$, q.e.d.

It should be observed here that the nature of $\left(\mathrm{S}^{\prime}\right)$ determines whether the pseudo-group will be finite or infinite dimensional. If the involutive prolongation of $\left(\mathbf{S}^{\prime}\right)$ is completely integrable the solutions will depend on a finite number of parameters, and for each $f^{1}$ the solutions to $\left(1^{\prime}\right)$ will depend on another finite system of parameters. Hence the pseudo-group will be finite dimensional, or a local Lie group. This is the case with the quaternions (see [4] for their system of equations). However, in general $\left(\mathrm{S}^{\prime}\right)$ need not be completely integrable in which case the pseudo-group will be infinite.

Using the lemma we shall now deduce necessary and sufficient algebraic conditions that $G$ satisfy (iii) and (iv) in the definition of pseudo-groups. Let $D_{j}$ be the $n \times n$ matrix each of whose elements is 0 except the $(j, j)$ th, which is 1 . Let $\varepsilon_{1 j}$ be the matrix obtained from the identity by interchanging the first and $j$ th rows. Let $\mathcal{F}$ denote the Jacobian $\left(\partial f^{i} / \partial x^{j}\right)$. Let $\alpha^{k}$ be the matrix $\left(a_{j}^{k h}\right) ; h, j=1, \cdots, n$; $k=2, \cdots, n$. Then equations $\left(1^{\prime}\right)$ may be written

$$
\mathcal{E}_{1 k} D_{k} \mathcal{F}=\mathscr{D}_{1} F Q^{k},
$$$$
k=2, \cdots, n \text {. }
$$

Suppose now that $f$ and $g$ are in $G$ with Jacobians $\mathcal{F}$ and $\mathcal{G}$, respectively. In order that $g \circ f$, with Jacobian $\mathcal{G F}$, be in $G$, it is necessary and sufficient that

$$
D_{1} \mathcal{F} Q^{k}=\mathcal{E}_{1 k} D_{k} \mathcal{G} \mathcal{F}=D_{1} \mathcal{G} Q^{k} \mathcal{F},
$$

or

$$
\mathfrak{D}_{1} \mathcal{G}\left(F Q^{k}-Q^{k} \mathfrak{F}\right)=0, \quad k=2, \cdots, n .
$$


Now, by the lemma, $g$ may be chosen so that the first row of $D_{1} G$ assumes at any given $\left(a^{1}, \cdots, a^{n}\right)$ any values except possibly those on a nowhere dense set. Hence

$$
\mathcal{F} \mathbb{Q}^{k}=Q^{k} \mathfrak{F}, \quad k=2, \cdots, n,
$$

must hold for every $f$ in $G$. We shall now determine conditions under which (3) occurs.

Let $f_{i}$ be a transformation in $G$ whose Jacobian satisfies, at $\left(a^{1}, \cdots, a^{n}\right)$

$$
D_{1} \mathcal{F}_{i}=\mathcal{E}_{1 i} D_{i}, \quad i=2, \cdots, n,
$$

which is possible unless the vectors which have 0 's except at the $i$ th components and 1 there, happen to fall in the distinguished nowhere dense subset of the lemma. In this case the reasoning still holds, except that one must replace the above functions by functions whose initial values differ from those prescribed above by arbitrarily small increments and then obtain the algebraic conditions on the $a_{j}^{\text {kh }}$ by a limit argument. For simplicity we shall assume the above functions are in $G$. Then

$$
\mathcal{E}_{1 j} D_{j} \mathcal{F}_{i}=\mathcal{E}_{1 i} D_{i} Q^{j}
$$

so

$$
D_{j} \mathcal{F}_{i}=\mathcal{E}_{1 j} \mathcal{E}_{1 i} D_{i} Q^{j}, \quad j=2, \cdots, n .
$$

Now, for any matrix $H, n \times n$,

$$
H=\mathfrak{D}_{1} H+\mathfrak{D}_{2} H+\cdots+\mathfrak{D}_{n} H,
$$

hence,

$$
\mathcal{F}_{i}=\varepsilon_{1 i} D_{i}+\sum_{j=2}^{n} \mathcal{E}_{1 j} \mathcal{E}_{1 i} D_{i} Q^{j}
$$

Then condition (3) for $\mathcal{F}_{i}$ at $\left(a^{1}, \cdots, a^{n}\right)$ may be stated

$$
Q^{k} \mathcal{E}_{1 i} D_{i}+\sum_{j=2}^{n} Q^{k} \mathcal{E}_{1 j} \mathcal{E}_{1 i} D_{i} Q^{j}=\mathcal{E}_{1 i} D_{i} Q^{k}+\sum_{j=2}^{n} \mathcal{E}_{1 j} \mathcal{E}_{1 i} D_{i} Q^{j} Q^{k},
$$

where $k=2, \cdots, n$ for each $i=2, \cdots, n$.

These are necessary conditions that $G$ satisfy axiom (iii). But conversely if $f$ is any element of $G$ with Jacobian $\mathcal{F}$ defined at a point $\left(a^{1}, \cdots, a^{n}\right)$, then

$$
D_{1} \mathcal{F}=\sum_{i=1}^{n} f_{i}^{1} \mathcal{E}_{1 i} D_{i}
$$


where $f_{i}^{1}=\partial f^{1} / \partial x^{i}$ evaluated at $\left(a^{1}, \cdots, a^{n}\right)$ is a scalar. Hence

$$
D_{j} F=\mathcal{E}_{1 j} D_{1} \mathfrak{F} Q^{j}=\sum_{i=1}^{n} f_{i}^{1} \mathcal{E}_{1 j} \mathcal{E}_{1 i} D_{i} Q^{j},
$$

$j=1, \cdots, n$. Moreover, equation (2) can be stated

$$
\mathfrak{D}_{1} \mathrm{Q}^{k}=\mathcal{E}_{1 k} \mathfrak{D}_{k}, \quad \quad k=2, \cdots, n .
$$

Hence,

$$
\begin{aligned}
\mathcal{F} Q^{k}-Q^{k} \mathcal{F}= & \left(D_{1} \mathcal{F}+\sum_{j=2}^{n} D_{j} \mathcal{F}\right) Q^{k}-Q^{k}\left(D_{1} \mathcal{F}+\sum_{j=2}^{n} D_{j} \mathcal{F}\right) \\
= & \sum_{i=1}^{n} f_{i}^{1}\left\{\left(\varepsilon_{1 i} D_{i}+\sum_{j=2}^{n} \mathcal{E}_{1 j} \mathcal{E}_{1 i} D_{i} Q^{j}\right) Q^{k}\right. \\
& \left.-Q^{k}\left(\varepsilon_{1 i} D_{i}+\sum_{j=2}^{n} \varepsilon_{1 j} \varepsilon_{1 i} D_{i} Q^{j}\right)\right\} \\
= & 0
\end{aligned}
$$

if $\left(3^{\prime}\right)$ holds. Hence $\left(3^{\prime}\right)$ is necessary and sufficient that axioms (iii) be satisfied, given axiom (ii). Now we shall show that axiom (iv) is a consequence of the others.

Let $f \in G$ with Jacobian $\mathcal{F}$. Let $h$ be the inverse of $f$ with Jacobian $\mathfrak{F C}$ so that $\mathfrak{F} \mathcal{H}=\mathscr{g}$, the identity.

Since (3) holds for $\mathcal{F}$, it obviously holds for its inverse at each point: FC $Q^{k}=Q^{k} \mathfrak{F}, k=2, \cdots, n$. If the identity whose Jacobian is $\mathscr{G}$, is in $G$, then $\varepsilon_{1 k} D_{k} \mathscr{G}=D_{1} \mathscr{I} Q^{k}=D_{1} Q^{k}$, so $\varepsilon_{1 k} D_{k} \mathfrak{H C}=D_{1} Q^{k} \mathcal{H C}=D_{1} \mathfrak{H C} Q^{k}$, $k=2, \cdots, n$. Hence $\mathfrak{H C}$ satisfies $\left(1^{\prime \prime}\right)$ and so $h$ belongs to $G$. To summarize:

TheOREM. Let $A$ be an $n$-dimensional algebra defined by $\pi_{j k}^{i}, i, j, k$ $=1, \cdots, n$, where the matrix $\left(\pi_{j k}^{1}\right)$ has an inverse, $\left(\rho^{i k}\right)$. Then define matrices $a^{k}, k=2, \cdots, n$, by $a^{k}=\left(a_{j}^{k h}\right)$ where

$$
a_{j}^{k h}=\sum_{i=1}^{n} \rho \stackrel{h i k}{k} \pi_{i j}
$$

$A$ necessary and sufficient condition that the local bianalytic transformations on $A$ which are $A$-analytic form a pseudo-group is that conditions (2) and (3') hold for these matrices $a^{k}$. Moreover, the closure of the pseudo-group under inverses follows from the other axioms.

Example. When $n=3$, 


$$
a^{2}=\left(\begin{array}{rrr}
0 & 1 & 0 \\
0 & 0 & 1 \\
2 & 1 & -1
\end{array}\right), \quad a^{3}=\left(\begin{array}{rrr}
0 & 0 & 1 \\
2 & 1 & -1 \\
-2 & 1 & 2
\end{array}\right)
$$

is an example of matrices satisfying the hypotheses.

\section{REFERENCES}

1. E. Cartan, Sur la structure de groupes infinis de transformations, Annales Scientifiques de l'Ecole Normale Supérieure vol. 21 (1904) pp. 153-206.

2. H. H. Johnson, On terminating prolongation procedures, Pacific J. Math. vol. 10 (1960) pp. 577-583.

3. M. Kuranishi, On E. Cartan's prolongation theorem of exterior differential systems, Amer. J. Math. vol. 79 (1957) pp. 1-47.

4. C. F. Moppert, On the notion of analyticity, Proc. Amer. Math. Soc. vol. 10 (1959) pp. 574-576.

Princeton University

\section{THE POLYNOMIAL DERIVATIVE AT A ZERO ANGLE ${ }^{1}$}

W. E. SEWELL

1. Introduction. The purpose of this note is to prove the following theorem:

Let $C$ be a piecewise analytic Jordan curve bounding the simply connected region $R$ and let $z_{0}$ be a corner of $C$ with exterior angle zero. Let the 1 st, $2 n d, \cdots$, th thential derivatives of the two arcs of $C$ at $z_{0}$ vanish. Let $P_{n}(z)$ be a polynomial of the degree $n$ in $z$ such that $\left|P_{n}(z)\right|$ $\leqq M, z$ on $C$; then $\left|P_{n}^{\prime}\left(z_{0}\right)\right| \leqq M K(\log n)^{1 / t}$, where $K$ is a constant depending only on $C$.

This problem has an interesting history. In 1889 A. Markoff ${ }^{2}$ proved that if $\left|P_{n}(z)\right| \leqq M,-1 \leqq z \leqq+1$, then $\left|P_{n}^{\prime}(z)\right| \leqq M n^{2}$. And since that time notable contributions are due to $M$. Riesz, S. Bernstein, G. Szegö, J. L. Walsh, and others. For example, if the exterior angle at $z_{0}$ on the curve $C$ of the theorem is $\alpha \pi$, G. Szegö $\ddot{o}^{3}$ has shown that for $0<\alpha \leqq 2$ we have $\left|P_{n}^{\prime}\left(z_{0}\right)\right| \leqq M K n^{\alpha}$. He has also shown that

Received by the editors March 29, 1960 and, in revised form, May 11, 1960.

1 This work is sponsored by the Office of Ordnance Research, U. S. Army.

2 On a problem of $D$. I. Mendeljeff (in Russian), Bulletin of the St. Petersburg Academy vol. 62 (1890) pp. 1-24.

- Über einen Satz von A. Markoff, Math. Z. vol. 23 (1925) pp. 45-61. 